\title{
Carboxylesterase-expressing Allogeneic Neural Stem Cells
}

National Cancer Institute

\section{Source}

National Cancer Institute. Carboxylesterase-expressing Allogeneic Neural Stem Cells. NCI

Thesaurus. Code C113657.

A preparation of allogeneic neural stem cells (NSC), derived from a human fetal cell line, that are adenovirally-transduced to express a modified form of the human enzyme carboxylesterase (CE) hCE1 m6, with potential adjuvant activity. Upon intracranial administration, NSCs localize to tumor sites, due to their tumor-trophic nature, and transiently express hCE1 m6. Intravenous co-administration of the prodrug irinotecan allows for the selective conversion by hCE1 $\mathrm{m} 6$ to its active metabolite and topoisomerase I inhibitor, SN-38, in the vicinity of tumor sites. This leads to a local antineoplastic effect and causes reduced toxicity and increased therapeutic efficacy of irinotecan. Since NSCs freely cross the blood-brain barrier, these cells can also be intravenously administered to target brain tumor cells. hCE1 m6 shows increased activity as compared to unmodified human CE. 